\title{
Catch the Bus: Probing Other-Than-Human Perspectives in Design Research
}

\author{
Viktor Bedö* \\ Critical Media Lab, Institute for Experimental Design and Media Cultures, Academy for Arts and Design, University of Applied \\ Sciences North-West Switzerland, Basel, Switzerland
}

Prompted by Catch the Bus, an experimental street game design project with and for autonomous buses, this study explores strategies to substantiate the speculation about other-than-human perspectives. It builds on philosophical arguments about the role of species similarity in grasping nonhuman experience and applies these arguments to thing perspectives. Gameplay and props from Catch the Bus instantiate a kind of similarity between human players and autonomous buses that emerges through the adoption of certain choreographies and sensing capabilities. The study contributes theoretical arguments to the debate of other-than-human perspectives in more-than-human design.

Keywords: street game design, thing perspective, species similarity, autonomous buses, embodiment, more-thanhuman design

\section{OPEN ACCESS}

Edited by:

Mattia Thibault,

Tampere University, Finland

Reviewed by:

Charlotte Magnusson,

Lund University, Sweden

Anton Nijholt,

University of Twente, Netherlands

${ }^{*}$ Correspondence:

Viktor Bedö

viktor.bedoe@fhnw.ch

Specialty section: This article was submitted to Human-Media Interaction, a section of the journal

Frontiers in Computer Science

Received: 30 November 2020 Accepted: 14 June 2021 Published: 12 July 2021

Citation:

Bedö V (2021) Catch the Bus: Probing Other-Than-Human Perspectives in

Design Research.

Front. Comput. Sci. 3:636107. doi: $10.3389 /$ fcomp.2021.636107

\section{INTRODUCTION}

What design research can gain from grasping other-than-human or alien perspectives is the ability to expand the range of beings and things that humans can engage with responsibly. Adopting alien or unfamiliar perspectives often demands a kind of probing. Space probes are sent out into unchartered territories to collect data samples from places no humans can reach. "Cultural probes" in social or design research provide designers with insight into other peoples' hopes and fears (Boehner et al., 2012). Probing takes up the challenge of adopting a vantage point other than one's own and thus engages with situations and places where the bodily or cultural presence of the researcher or designer is challenging or impossible. The challenge of exploring perspectives that emerge from experiences, interactions, and entanglements that are different from the designer's experiences is not new. When probing other-than-human perspectives, however, this endeavor is extended to beings or things that have ascribed the agency to engage with the world based on their material specificity and specific locale, but not primarily based on discursive, cultural, or cognitive terms. Imagining what the world is like for nonhumans-for example, autonomous buses-thus involves a fair amount of fiction or speculation.

Depending on the desired epistemic outcome of design exploration, the design researcher may attempt to substantiate speculation with something that is grounded in experience. Gameplay or playful enactment can seamlessly overlay everyday life with fiction and thus is a fertile ground for balancing everyday experiences with speculation. Street games in particular are embedded in specific urban contexts so that the mechanisms of the city and the rules, props, and fiction of the games merge to form a coherent experience in gameplay, rendering them in situ laboratories for alternative or future imagination of the city (Bedö 2019). The street game designer balances the amount of fiction or imagination that is injected into the experience of a site during gameplay with real-life variables, such as pedestrian flows, technology use, or traffic. Thus, gameplay can be used as a strategy to have 
players adopt alien perspectives by placing them at alien sites, equipping them with alien capacities, and framing alien entanglements with other beings and things.

Expanding on Catch the Bus, an experimental street game design project, this study investigates the potential strategies and limitations of adopting the perspective of an autonomous bus. ${ }^{1}$ The creative process of the Catch the Bus project resulted in a design artifact and playful interactions which triggered this study's reflections on nonhuman perspectives. The ambition to engage with an other-than-human perspective through playfully enacting an autonomous bus was implicitly present throughout the design process. Grasping the perspective of another being or a thing foregrounds more fundamental epistemological dimensions of design research. This study attempts to make modes of engagement with nonhuman perspectives explicit by relating arguments from ontology, philosophy of the mind, and nonhuman turn debates to the street game design project. The argument develops based on an interpretation of perspective that emphasizes a being's or thing's material/bodily and sensory constitution and unique position from which entanglements with other things emerge. The study explores the role of similarities in bodily and material constitution, size, shape, and sensing capability as central aspects in substantiating the adoption of alien perspectives. With this, the study contributes to the theoretical backdrop for explorative methodologies of designing for humans and autonomous things.

\section{CATCH THE BUS PROJECT}

The following section briefly introduces the Catch the Bus project's context, the design process, and some of the games that emerged from it. The project took place in the private road network of the Charité Hospital campus in Berlin Mitte where the Berlin public transport company (Berliner Verkehrsbetriebe, BVG) operates one of its prototype autonomous bus services. The buses are stage $3+$ autonomous vehicles which means that they autonomously follow a preprogrammed route and carry passengers, but they still require monitoring and occasional interventions by a human operator who is present on the bus at all times. The tasks of the operators-who in this case were former BVG bus drivers-include releasing the emergency brake when necessary and manually maneuvering via a portable control panel around parked cars that obstruct the path of the bus. Our contacts from the BVG supported our explorations and design process by granting us access to the buses both within and outside of regular operational times and introduced us some aspects of the technology which are not accessible to the wider public.

\footnotetext{
${ }^{1}$ Catch the Bus is a 2019 project with and for autonomous buses by Simon Johnson (Cofounder of Free Ice Cream: http://freeicecream.co.uk) and myself (Tacit Dimension: http://tacitdimension.com and Critical Media Lab Basel: https:// criticalmedialab.ch), commissioned by the Fiction Forum in Berlin. Fiction Forum is a 2019 initiative of the German Federal Ministry for Economic Affairs and Energy with the mission to make visible creative industries' economic, societal, and innovative impact.
}

Project duration was 10 days, including an exploration phase, game design, a public playtesting session, a public game release session, and a panel discussion about mobility. In the first two days of the project, we undertook exploratory design research which included familiarizing ourselves with the BVG pilot project and the operation of the autonomous buses during a guided tour, by interacting with the bus itself. We investigated the behavior of the bus by triggering emergency brakes in different ways (we later promised the BVG not to include jumping in front of the bus as a game mechanic) and overtook the bus on foot and on bikes to explore what makes it slow down or stop. We observed how pedestrians who were too close to the curb slowed down the bus or brought it to a halt, which happened regularly at narrow sidewalks. We spoke to the bus operators about their tasks and responsibilities, learning that, as an act of courtesy, they sometimes switch to manual mode when approaching an intersection to signal to an approaching car that they will give way. Finally, we talked to passengers to learn about their level of trust in the autonomous buses.

After the exploration, we created game prototypes which we tested in a public playtest. The playtest session took place on the fifth day of the project with testers from Fiction Forum and the Berlin street game design community. For the playtest, we arranged four game prototypes in a game tour around the Charité campus. We moderated the session by setting up games, guiding the players through the tour, and introducing the rules of each game. After the playtest session, we iterated the games based on player feedback and our own observations and finalized some of them for the public release event. This took place on the last day of the project with a game tour featuring three games, two of which are outlined below. In addition to the communities who took part in the playtest, the Fiction Forum's stakeholders from the Ministry of German Federal Ministry for Economic Affairs and Energy and BVG joined in for the game release event.

We approached the design process with initial questions about care, sensing capabilities, and world-making of the buses, but we did not hold these directions strongly and allowed the research and design process to be guided by unexpected cues in the field. The aim to mimic the operational principles of an autonomous bus in the game was present very early in the project. To better understand how an autonomous bus engages with the city and traffic, one of the first design commitments in the project planning phase was to build our own autonomous bus comprising low-tech sensing, wet intelligence (players), and muscle-propelled movement. This "toy bus" was realized in the form of a metal frame (in BVG yellow) with the footprint of the real autonomous bus and equipped with proximity sensors (Figure 1).

A prominent feature that the toy bus emulated from the BVG bus was its ability to sense the environment. The BVG bus's key sensing instruments are light detection and ranging (LIDAR) sensors, with $3 \mathrm{D}$ sensors to the front and back and 2D sensors on all four corners. The bus essentially follows virtual tracks manually hard-wired into the map software of the bus, so the LIDARs provide sufficient sensing for the bus to slow down or stop if objects such as falling leaves or dense rain obstruct its path 


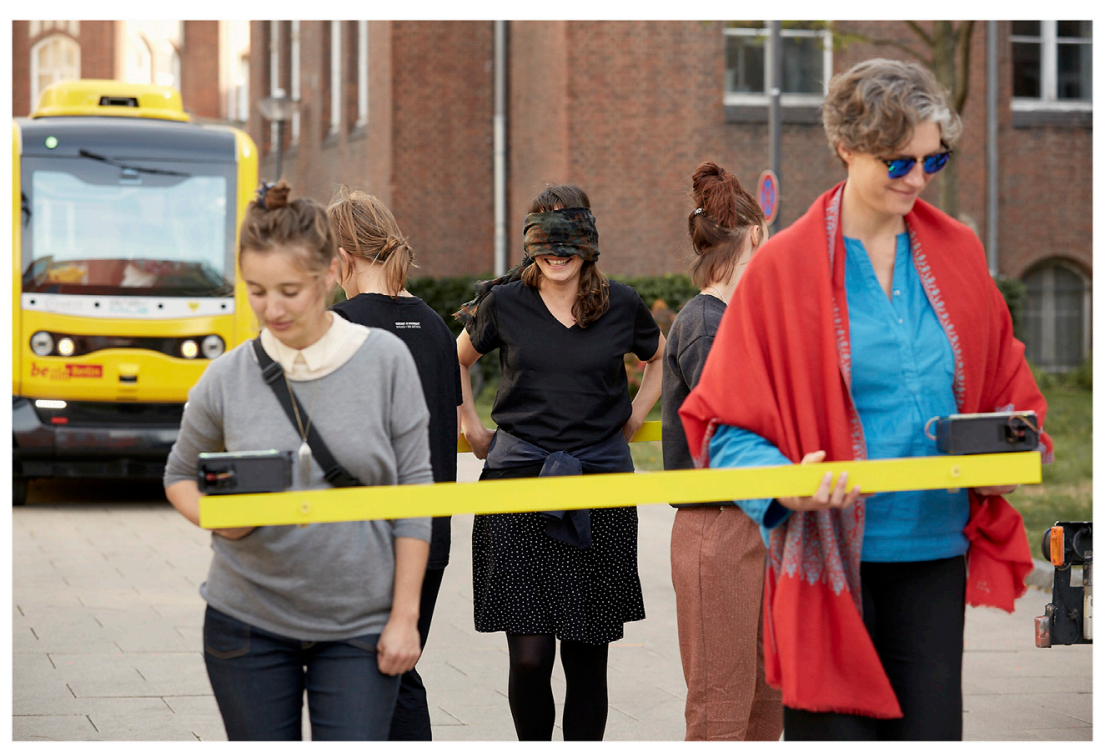

FIGURE 1 | Toy bus with players.

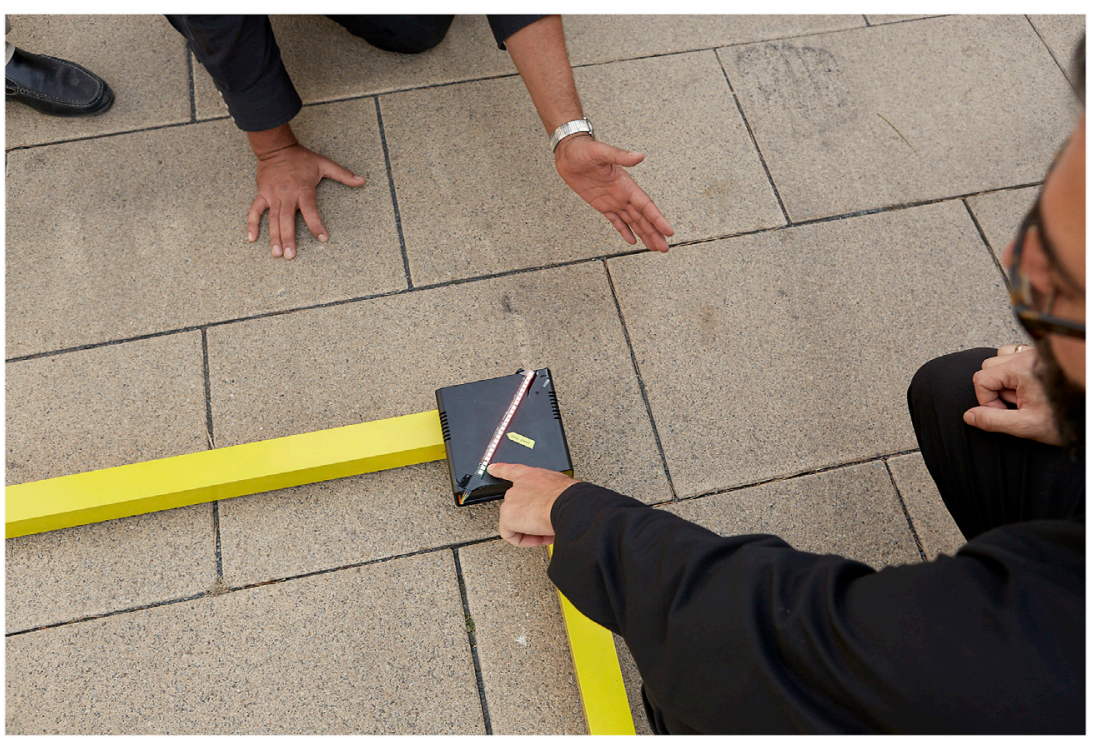

FIGURE 2 | Proximity sensor with display.

or come too close. Although the BVG buses are equipped with cameras, they were not in use and were even covered with duct tape by the time the project took place.

Based on that, we equipped the rectangular metal frame of the toy bus with proximity sensors and set up minimalist LED-based displays in all four corners (Figure 2). The distance sensors were directed outwards at an angle of $135^{\circ}$ from the four corners of the frame and measured the proximity of objects in a range of $0-4 \mathrm{~m}$ (Figure 3). On the housing of the sensors, we mounted LED light strips as simple displays showing the distance to objects around the frame. The lights gradually turned from green to red when something came close to the frame. As well as on this gradual scale, there were two marks, one for "close" when objects were within a $2 \mathrm{~m}$ " range and another for "too close" when objects were within $0.5 \mathrm{~m}$. These settings imitated the behavior of the BVG bus, which slows down if something gets within a range of $1.5 \mathrm{~m}$ and comes to a halt at a range of $0.5 \mathrm{~m}$. The buses turned out to be defensive with an extremely low-risk affinity, an observation that was later clearly confirmed by the BVG as one of the current core design principles.

During gameplay, players stepped inside the frame of the toy bus and carried it by holding it. Four "sensor players" were positioned in each corner of the toy bus at the sensor boxes with the display. A blindfolded "steering algorithm" player held on to 


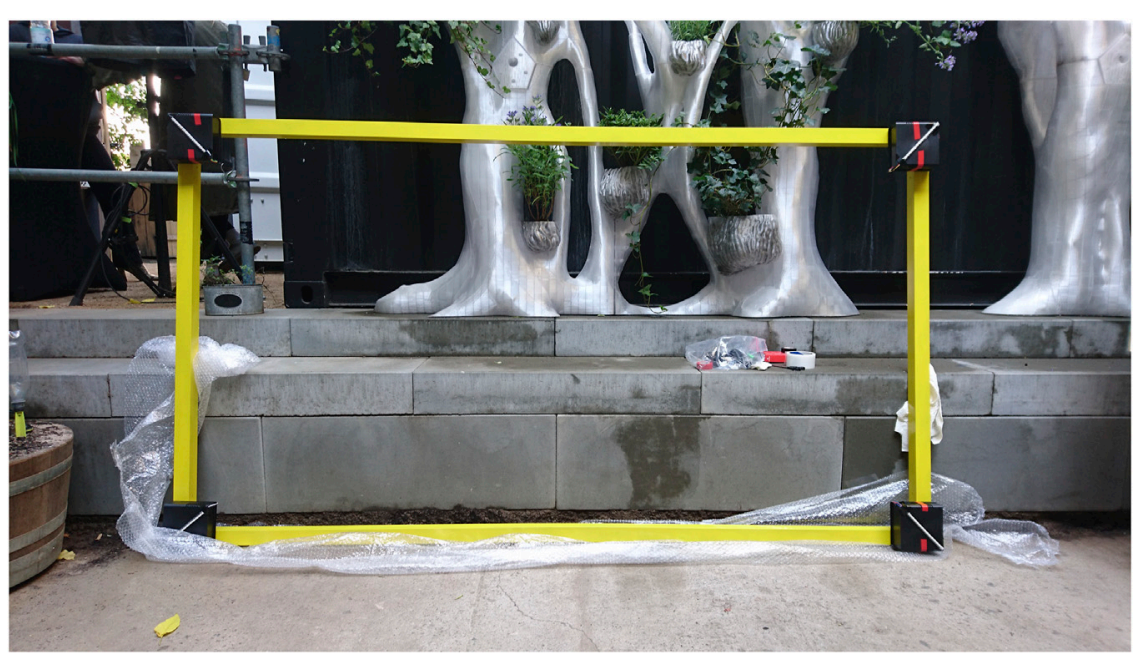

FIGURE 3 | Toy bus.

the back of the toy bus and steered it left or right. The sensor players were responsible for communicating the sensor readings on the display to the steering algorithm player who was navigating. Forwarding the sensor readings to the blindfolded steering algorithm turned out to need a fairly high level of coordination. Only after a learning curve of one or 2 min did a protocol emerge of calling out sensor readings in a way that the steering algorithm could correctly interpret. Even after establishing this protocol, the toy bus continued to operate with some lag. This lag and the process of establishing and iterating the protocols for coordinating between sensor players, the steering algorithm, and objects in the environment turned out to be the strongest mechanic in the gameplay.

\section{THE GAMES}

In this section, I will introduce two of the games that were played in the final release of Catch the Bus so that the specificities of the game mechanics and the playful interaction between players, game props, and the environment can stand as a heuristic device for the arguments about adopting nonhuman perspectives that follow in Probing Alien Perspectives and Like a Bus.

\section{Invisible Map}

We named this game after a map that remained invisible to some players during gameplay. In the Invisible Map, besides the players operating the toy bus ("sensors" and steering algorithm), we introduced the "environment" player-type. The main mechanic of the game is that the toy bus navigates a path and tries to avoid collisions with the environment, namely, players who embody objects in the environment alongside the path of the bus. The Invisible Map is a cooperative game where the toy bus players and the environment players work together to get the bus from Bus Stop 1 to Bus Stop 2.
A standard path between Bus Stop 1 and Bus Stop 2 was approximately $50 \mathrm{~m}$ long and several paths were marked out on the ground by tape dots (Figure 4). Environment players would stand on the dots to create the boundaries of the path and guide the toy bus. For replayability, we overlayed several paths on the ground using different colors. Only environment players knew the color of any current round so that the bus players were not able to effectively navigate based on the colored dots and had to rely on the environment. For complexity, we also added forks in the path, opening up alternative routes. The width of the path was approximately $2-2.5 \mathrm{~m}$, approximately 1.5 times the width of the bus body so that it was challenging but possible for the bus to move on the path flanked by the environment. The distance between the dots measured approximately one step, dense enough that the toy bus could not slip between two environment players on one side of the path but rare enough that the environment did not too obviously delineate the path ahead for the sensor players.

According to the game fiction, the environment players are "downloaded in real time on the map": they stand on the marks on both sides of the path, and when the back of the toy bus passes an environment player, the player moves quickly to take up their place at the front of the line (Figure 5). The environment players form a dynamic urban landscape that flanks the path (for which a minimum of four environment players are needed) and which the toy bus can sense (Figure 6).

The goal of the game is for the toy bus and the environment to cooperate in allowing the toy bus to travel from one bus stop to the other. If the bus hits an object in the environment (player or nonplayer) - that is, if the reading on any of the sensor boxes shows "too close"-everybody loses a point. Players succeed in the game if the toy bus arrives at the goal bus stop without losing more than three points. If the bus hits an object more than three times, the autonomous bus does not pass the road safety standards test and everybody loses. 


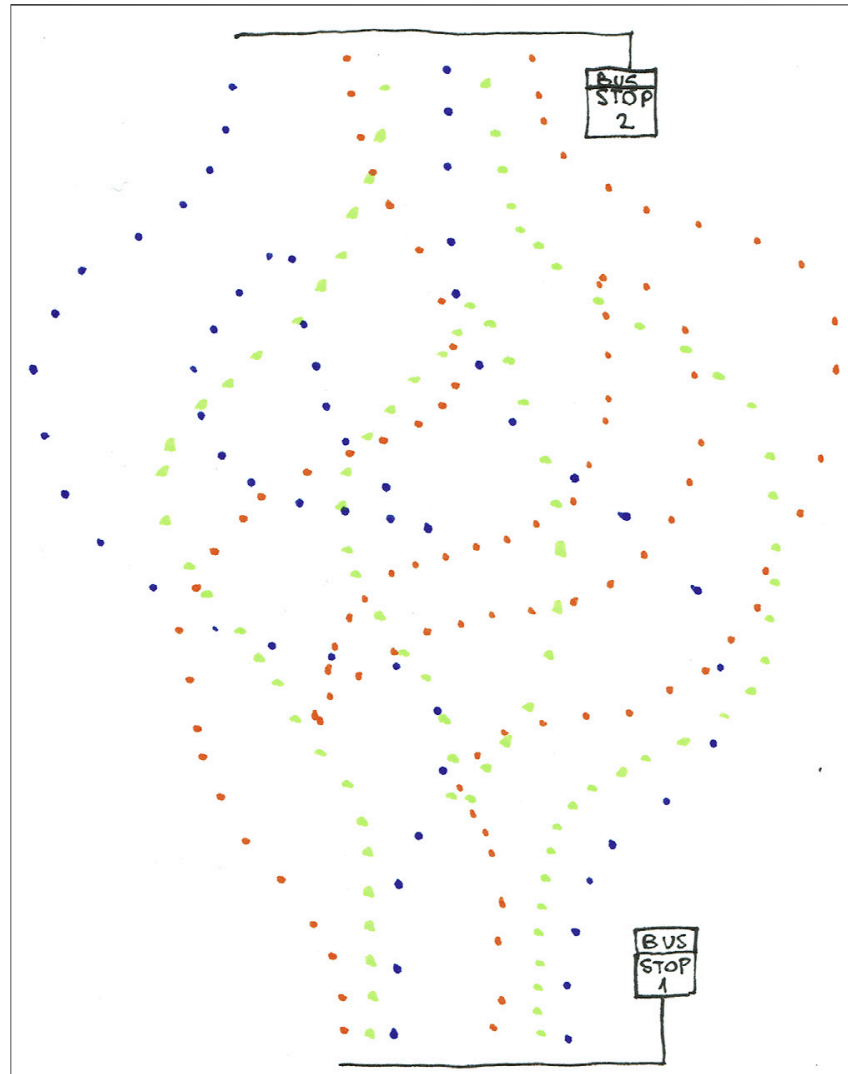

FIGURE 4 | Invisible Map.

The player experience turned out to center on the dynamic attunement between sensors, steering algorithm, and environment. Each became attuned to the other in the cooperative game setting, as they pulled each other toward Bus Stop 2. We even could have introduced environment in the game fiction as something internal to the bus's navigational mechanisms, such as "map data," without changing the interplay between the players, which hints at the blurring of the perceptive and proprioceptive aspects of navigation. This interplay between bus players and the environment players was a sound instantiation of the situated entanglements of an autonomous bus with its environment and thus created a unique bus perspective relative to its position and bodily and sensory constitution.

\section{The Overtake}

It is worth to briefly mention another game from the release, where the sensing and steering mechanisms of the toy bus were the same as in the Invisible Map, but the toy bus interacted with the real BVG bus instead of an environment constituted by players. The goal in The Overtake was to overtake the BVG bus with the toy bus without bringing the BVG bus to a halt. The "bus" players' setup in the toy bus was identical to the Invisible Map setup, with four sensor players and one blindfolded steering algorithm. The challenge was to get close enough to the BVG bus during the maneuver so that it slowed down but not so close that it would come to a halt (Figure 7). In this maneuvering game, the sensing and steering mechanisms of the two busses entered into negotiations to keep moving safely. The challenge for the toy bus was to coordinate the movement of both buses while adjusting to the road conditions and environment. To succeed, the toy bus needed to become attuned to both the street and the real autonomous bus (Figure 8).

\section{PROBING ALIEN PERSPECTIVES}

Perspective in the context of this study refers to the distinctive entanglements which emerge from the unique position, history, and momentary bodily engagement of beings and things with others in their more or less immediate proximity. Grasping the perspectives of other people is less about reading their mind than about understanding with whom and what they interact in a given moment and how these interactions are rooted in their unique life experiences, attitudes, and abilities. Likewise, grasping a bus's perspective is not necessarily about un-black-boxing or reverseengineering the algorithms of an autonomous vehicle, but rather about the unique relations it enters into with other things in its proximity during a specific moment and place: interactions with pedestrians, traffic, its operator, entanglement with regulations, or operational mechanisms that emerge through its material specificities (such as its algorithms, materials, dimensions, and sensing capabilities). For design research, in the sense of generating knowledge that informs better designs, the interest around grasping a nonhuman perspective is primarily epistemological. Ontological questions such as whether intelligence, mind, or sentience are qualities that the bus possesses have implications for the politics of design but are outside the scope of this study.

Although the Catch the Bus games do not share many similarities with cultural probes in their use and mechanisms, they share one key affordance, namely, enabling the adoption of unfamiliar and otherwise inaccessible perspectives. Cultural probes are research tools that design researchers or social scientists use to learn about other people's lives, kits that are sent out to people to use in their everyday life before sending them back. Kits might contain tools for recording and marking, for example, a camera, a notebook, a calendar, or a map, as well as a set of instructions on how, where, and when to use these tools. The users of the kit would take photos in specific situations or about a specific object, markdown feelings, encounters, or any kind of notes guided by the instructions which come with the kit. The instructions are worded in such a way that they constrain the topics the probe addresses and encourage the answers to be formatted in a certain way, while at the same time allowing for some degree of openness and improvisation (Boehner et al., 2012). Graver and Dunne, who coined the term cultural probe, conceived it as an explorative and playful tool to understand people and settings, "[making] a virtue of uncertainty and risk, acknowledging and celebrating the idiosyncratic interpretations of designers and participants." (Boehner et al., 2012, p185). When researchers cannot follow the same trajectories and engage with the same things or beings as the people whom they would like to 


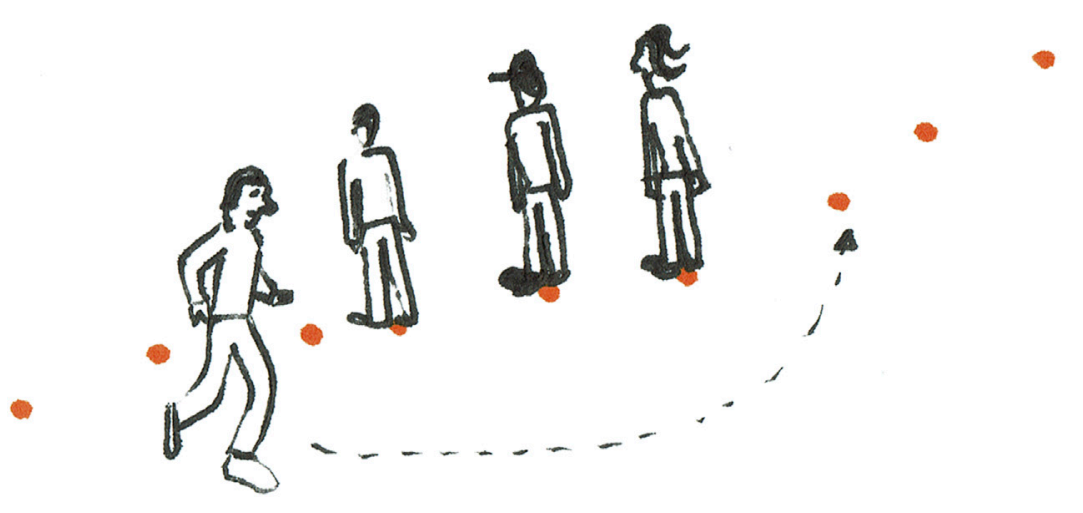

FIGURE 5 | Dynamic urban landscape.

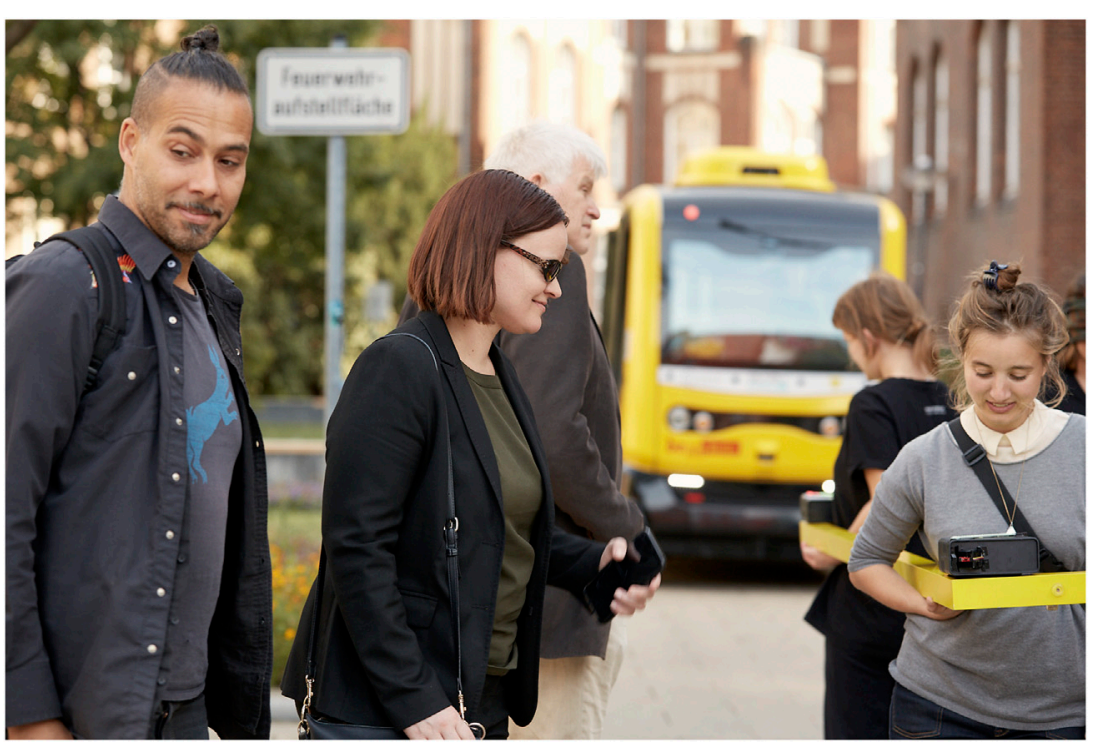

FIGURE 6 | The toy bus in the environment.

learn more about, they rely on probing how their research subjects are affected by certain events and encounters that materialize on those trajectories. The further apart the life experiences of a researcher are from the other person's experiences, the more they have to rely on instruments of probing to develop a grasp of what it is like for the other person. Also, with increasing distance, the interpretation of the data returned by probes increasingly turns speculative, in the sense of not being grounded in one's own experiences.

In adopting alien perspectives, such as a "thing perspective," speculation of some kind seems to be the only option. Speculating about an alien or other-than-human perspective means building to some extent on plausible human knowledge about the kind of entanglements a nonhuman being or thing is embedded in, but applying fiction wherever this knowledge thins out. The podcast
"Everything is Alive"2 features interviews with things such as sand, a bar of soap, and a subway seat. In the podcast, we learn about their life, their joys, fears and desires, and connections both with other things and with humans. The things are impersonated by actors, who use the improv theatre method for the unscripted interviews. The human impersonating sand builds on their obviously limited access to how the world is for sand, and their knowledge about how sand is handled, used, transported, and its value in production processes combined with a huge amount of imagination, allows plausible, although fictive and speculative, immersions into the sand's perspective.

${ }^{2}$ https://www.everythingisalive.com. 


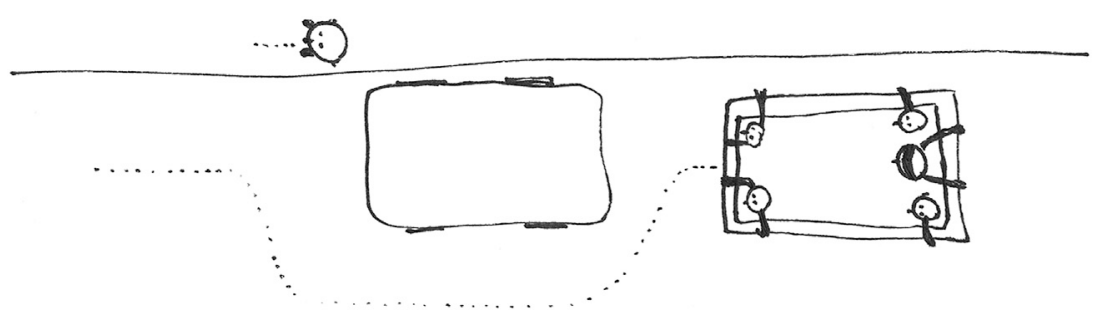

FIGURE 7 | The overtake maneuver.

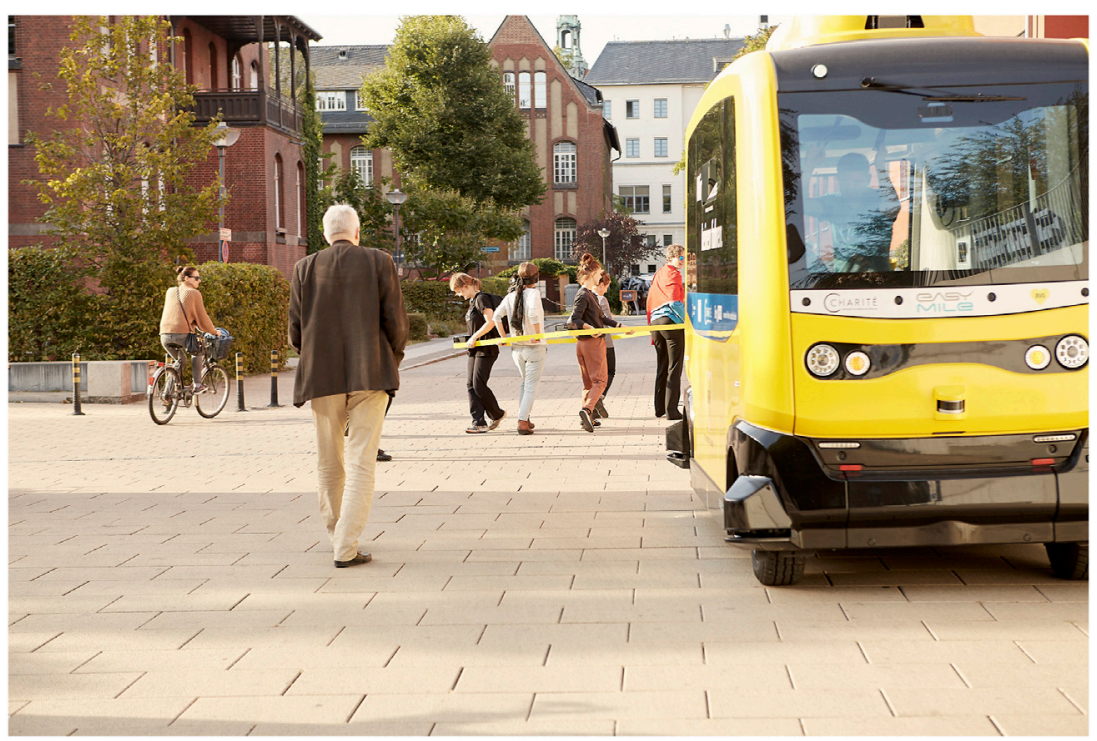

FIGURE 8 | The overtake.

Another point of reference for adopting other-than-human perspectives is the design method "Interview with Things" (Chang et al., 2017; Reddy et al., 2021). Similar to the "Everything is Alive" interviews, it builds on impersonating things in interviews to explore everyday sociomaterial networks in which the things are operating. However, as a design research method, its ambition is to substantiate the speculation with experiential data as much as possible. To this end, the Interview with Things method seeks to reduce the amount of speculation in the interview by finding ways of extracting information and knowledge from the situated and material contexts of the interviewed thing and applying them during the interview. In an instance of employing the Interview with Things method for exploring urban scooters in Taipei, design researchers mounted cameras and sensors on the scooters to capture photo and video footage and sensor readings. The researchers then preprocessed this data to make it available to the actors, who studied and internalized the materials in preparation for the interviews.

Certainly, many more projects could instantiate speculative strategies when engaging with unfamiliar and alien perspectives. The aim here was merely to illustrate the principles of blending empirical knowledge, data gathering, and fiction in speculating about thing perspectives in design research. Adopting other people's perspectives can be challenging enough; adopting the perspectives of nonhuman beings is, some might say, impossible or fictional altogether. The epistemological question to explore for design research remains: What are the fruitful pursuits for strengthening experiential and/or embodied knowledge in the speculation about nonhuman perspectives?

\section{LIKE A BUS}

To highlight the key role of similarity with nonhumans in the ability to adopt nonhuman perspectives, this section looks at experiments and theoretical arguments about the possibility of grasping nonhuman experience by enacting the situatedness of nonhumans and placing oneself within nonhuman niches. I will suggest that the Catch the Bus project probes the capacity of such similarity by placing players in traffic, choreographing them into adopting the physical footprint of an autonomous bus, and modulating their sensory and navigational data processing capacities so that they mimic the sensing and processing capacities of the bus.

Adopting a nonhuman perspective by temporarily sharing the environmental niche of another species was attempted by ex-vet and barrister Charles Foster (2016) who committed to living like a 
badger, an otter, an urban fox, a deer, and a swift, each for a short amount of time. During the attempt to live like an urban fox, for example, he foraged in bins and slept in gardens. In a similar vein, designer Thomas Thwaites (2016)joined goats in the Alps. He took the experiment even further by modifying body posture and locomotion using a prosthetic exoskeleton, to approximate a goat-like physicality. Both Foster's and Thwaites's ambitions were to better connect to the animal world by experiencing what the world is like for animals. When reflecting on Fosters's and Thwaites's experiments from a performance studies perspective, Orozco and Parker-Starbuck not only point out how the playfulness of pretending allows a different relationship to the animals (Orozco and ParkerStarbuck 2017, p64), but emphasize the primacy of a physical approach over a discursive one. Referring to Foster and Thwaites, they argue that situating embodiment is a more authentic way of apprehending the nonhuman than rational scientific discourses and welcome the "favouring of embodiment [as] recognition of the opening that performance, pretense and mimicking bring about as a potential reaching out towards the animal" (Orozco and ParkerStarbuck 2017, p64). An interpretation of perspective that emphasizes a being's or thing's unique position and material/bodily and sensory constitution is more invested in prediscursive, prelingual, or precognitive aspects of engaging with nonhumans. This prelingual, preconceptual, prediscursive, and pre-emotional mode of interacting or intra-acting with other things constitutes what Massumi describes as the space of effect (Massumi, 2015), and Shaviro describes as sentience (2015). In this sphere, the entanglements of things with other things emerge from their material specificities, unique positions, and their proximity to each other. Adopting a perspective in this sense means engaging with the things that the other is engaging with and engaging in similar ways, which in turn shifts resemblance and similarity into focus.

Prediscursive strategies are central for the adoption of nonhuman perspectives not only because we cannot ask an urban fox about their experience of the urban or ask a bus about its experience of traffic. Through the ability to speak, we can share to a much greater extent "what it is like" for other humans. But the private and public aspects of a human or nonhuman entity always go together, and ultimately, we always lack full cognitive access to other humans' inner experience: "What David Chalmers calls the 'hard problem' of consciousness indeed plays out the same way in relation to a bat or a cat-or for that matter, in Chalmers's notorious example, to a thermostat-that it does in relation to another human being" (Shaviro 2015, p29), and although we have a better grasp of other humans than we do of nonhumans such as animals, for example, a more extensive grasp of another being's experience based on species similarity is only a difference in degree and not in kind (Shaviro 2015, p29).

Species similarity was raised most prominently as a key discussion point in grasping nonhuman points of view or experience by philosopher Thomas Nagel in "What Is It Like to Be a Bat?" (1974). His core argument was that because of a different sensory and bodily setup, humans are not able to grasp what the world is like for bats (i.e., bat experience), and thus, it is impossible to know as humans whether bats have consciousness at all. For the philosophy of mind, Nagel's ontological question of whether bats have minds is of key interest. However, the relevance for design research is in the epistemological implications of how Nagel builds his arguments. Nagel's choice of bats as a case in point for his argument zeroes on a sphere where the similarity between humans and other species becomes elusive enough to support the problematization of adopting a nonhuman perspective: "I have chosen bats instead of wasps or flounders because if one travels too far down the phylogenetic tree, people gradually shed their faith that there is experience there at all" (Nagel 1974, p438). Nagel elaborates on how bats precisely discriminate between distance, size, shape, motion, and texture of things by echolocation, which is based on how objects reflect the bats' high-frequency shrieks. He argues that this form of perception is so different in its operation to human senses that humans can neither experience nor imagine what it is like to be a bat. Nagel poses the question of what method could possibly permit us to extrapolate the inner life of the bat from our own experience:

"Our own experience provides the basic material for our imagination, whose range is therefore limited. It will not help to try to imagine that one has webbing on one's arms, which enables one to fly around at dusk and dawn catching insects in one's mouth; that one has very poor vision, and perceives the surrounding world by a system of reflected high-frequency sound signals; and that one spends the day hanging upside down by one's feet in an attic. In so far as I can imagine this (which is not very far), it tells me only what it would be like for me to behave as a bat behaves. But that is not the question. I want to know what it is like for a bat to be a bat. Yet if I try to imagine this, I am restricted to the resources of my own mind, and those resources are inadequate to the task. I cannot perform it either by imagining additions to my present experience, or by imagining segments gradually subtracted from it, or by imagining some combination of additions, subtractions, and modifications. To the extent that I could look and behave like a wasp or a bat without changing my fundamental structure, my experiences would not be anything like the experiences of those animals. [...] Even if I could by gradual degrees be transformed into a bat, nothing in my present constitution enables me to imagine what the experiences of such a future stage of myself thus metamorphosed would be like" (Nagel 1974, p439).

Nagel delivers the argument that on grounds of the constitution of human bodies and sensing capabilities, it is impossible to extrapolate from human experience the experience of a bat; in other words, certainty about the existence of bat consciousness is beyond the epistemological horizon of any human being. At the same time, with his argument, he also outlines the path to the grasping of bat experience, which although we never pursue entirely, we can partially explore for a better grasp of nonhuman perspectives.

We see how with decreasing similarity in sensory and bodily constitution, humans' ability to take the perspective of other lifeforms decreases too. Being confronted with decreasing similarity with the nonhuman, as Shaviro points out in reference to Nagel's argument, "[ $t]$ he best we can do is to create metaphors and similes-or as I would rather say, esthetic semblances-that allude in some way to chiropteran or canine existence" (Shaviro 2015, p26). While the challenge of grasping other humans' unique perspectives emerges through differences in culture and personal histories (a challenge that is addressed by cultural probes), grasping perspectives of very different beings, such as ants, or even things, such as thermostats, is always in 
a sense speculative. When similarity in bodily and sensory makeup ceases to support the epistemology of grasping other-than-human perspectives, it is metaphors, speculation, and fiction that start to take over. However, strategies to increase similarity offer a means by which to achieve a less arbitrary and anthropomorphistic grasp of other-than-human perspectives.

During gameplay in the Catch the Bus project, players move among other vehicles in a metal frame which has the footprint of a small autonomous bus. They rely on distance sensors for sensing and navigating the environment, resembling the sensing and navigating principles of the autonomous bus's LIDAR system. The similarity of the toy bus with the original autonomous bus is evidently imperfect, partial at best. However, players who were partaking in the Catch the Bus games most likely displayed the highest level of similarity to an autonomous bus as ever before.

\section{CONCLUSION}

In design research, exploratory prototypes turn into speculative design artifacts when the design proposals they embody address future or alternative realities, with diminishing similarities to the contemporary context. Design exploration and testability gradually give way to fiction. It is in the hands of the design researcher to create a balance between prototyping and speculation based on the epistemological ambitions of a project. Speculative design is a powerful approach to creating imaginaries about desirable futures or scrutinizing dystopian extrapolations of our present. At the same time, an increasing degree of speculation detaches the design artifacts from their anchorage in everyday experiences. This shift from prototyping to speculation is somewhat analogous to the increasing level of speculation in adopting alien perspectives: with decreasing similarity of the beings or things whose perspective the human designer aims to grasp, the degree of fiction increases in the speculation about their perspective. If the general ambition of a design researcher's exploration is to generate substantiated design knowledge about the way a thing engages with other things, reducing the degree of fiction becomes the prime challenge. Game design manages this by merging fiction and prop enactment with real urban mechanisms.

The Catch the Bus project's scope of playfully exploring how people, traffic, and autonomous busses engage with each other did not allow for in-depth research about insights and knowledge that the experience generated for participants. But it generated a setup for shifting perspectives that is arguably a source of new knowledge. It is evident that enacting an autonomous bus as a collective of players can only provide a highly fictitious grasp of what it is like to navigate traffic as a bus. However, any increase of similarity to the build and situatedness of an autonomous bus carries the promise of increasing the authentic grasp of an autonomous bus's perspective. Performing and mimicking nonhumans does not mean that humans turn into nonhumans, but allows for successful strategies of turning to nonhuman beings (Orozco and Parker-Starbuck 2017, p67).

Tapping into philosophical debates about the nonhuman turn and design research, this study argues that adopting some degree of similarity in bodily and sensory makeup becomes key in substantiating the speculation about nonhuman perspectives. In Donna Haraway's "Camille" stories, the reader witnesses how more-than-human symbionts emerge over five generations as a deliberate strategy to better "live in intimate and worldly care-taking symbiosis with another animal as a practice of repairing damaged places and making flourishing multispecies futures" (Haraway 2016, p146). As a symbiont who links a human body to a monarch butterfly, Camille has vibrant orange and black skin and altered sensing capabilities provided by butterfly antennae. Mimesis is not the ultimate point of the alterations in Haraway's speculative fabulation; instead, species similarity becomes a central strategy in extending what she calls "respons-ability" to the more-than-human realm: "[The symbionts] roles in the symbioses were to teach and to flourish in every way possible in dangerous and damaged times" (Haraway, 2016, p147). The Catch the Bus project gives an instance of an exploratory design research approach for decentering from the human perspective. It opens up the possibility of generating design knowledge from a nonhuman vantage point while incrementally shifting the weight from fictions and metaphors to embodied experience. Any movement in this direction reduces the weight of fiction and, with this, the pitfalls of anthropomorphizing the bus perspective. The combination of playfulness-merging the real and fictive-and probing-shifting away from one's own perspective to otherwise inaccessible perspectives-is the basis of its epistemological export for design exploration.

\section{DATA AVAILABILITY STATEMENT}

The original contributions presented in the study are included in the article/supplementary material; further inquiries can be directed to the corresponding author.

\section{AUTHOR CONTRIBUTIONS}

The author confirms being the sole contributor of this work and has approved it for publication.

\section{FUNDING}

The writing of this study was supported by the Swiss National Science Foundation (grant number 175913).

\section{ACKNOWLEDGMENTS}

I thank Simon Johnson with whom I created the Catch the Bus games and who came up with the idea of the toy bus in the first place. Also, I thank Jennifer Aksu and the whole Fiction Forum team for inviting Simon Johnson and me to create this project and for their support in producing it. I am grateful to Iohanna Nicenboim for her comments about the main arguments of this study. 


\section{REFERENCES}

Bedö, V. (2019). "Rapid Street Game Design: Prototyping Laboratory for Urban Change," in The Hackable City (Singapore: Springer), 51-65. doi:10.1007/978-981-13-2694-3_3

Boehner, K., Gaver, W., and Boucher, A. (2012). "Probes," in Inventive Methods: The Happening of the Social. Editors C. Lury and N. Wakeford (Abingdon: Routledge), 185-201.

Chang, W. W., Giaccardi, E., Chen, L. L., and Liang, R. H. (2017). "“Interview with Things" A First-Thing Perspective to Understand the Scooter's Everyday SocioMaterial Network in Taiwan," in Proceedings of the 2017 Conference on Designing Interactive Systems, 1001-1012.

Foster, C. (2016). Being a Beast: Adventures across the Species divide. MacmillanNew York, NY.

Haraway, D. (2016). Staying with the Trouble: Making Kin in the Chthulucene. Duke University Press Durham.

Massumi, B. (2015). “The Supernormal Animal," in Nonhuman Turn. Editor R. Grusin(Minneapolis: University of Minnesota Press Minneapolis), 1-18.

Nagel, T. (1974). What Is it like to Be a Bat?. Phil. Rev. 83, 4435-4450. doi:10.2307/ 2183914

Orozco, L., and Parker-Starbuck, J. (2017). Goats, Badgers and Other Beasts. Perform. Res. 22 (2), 63-68. doi:10.1080/13528165.2017.1315972
Reddy, A., Kocaballi, B., Nicenboim, I., Søndergaard, M. L. J., Lupetti, M. L., Key, C., et al. (2021). "Making Everyday Things Talk: Speculative Conversations into the Future of Voice Interfaces at Home," in Extended Abstracts of the $2021 \mathrm{CHI}$ Conference on Human Factors in Computing Systems (Yokohama, Japan. ACM, New York, NY, USA: CHI '21 Extended Abstracts).

Shaviro, S. (2015). "Consequences of Panpsychism," in Nonhuman Turn. Editor R. Grusin(Minneapolis: University of Minnesota Press Minneapolis), 19-44.

Thwaites, T. (2016). GoatMan: How I Took a Holiday from Being Human. Chronicle Books London.

Conflict of Interest: The author declares that the research was conducted in the absence of any commercial or financial relationships that could be construed as a potential conflict of interest.

Copyright (C) 2021 Bedö. This is an open-access article distributed under the terms of the Creative Commons Attribution License (CC BY). The use, distribution or reproduction in other forums is permitted, provided the original author(s) and the copyright owner(s) are credited and that the original publication in this journal is cited, in accordance with accepted academic practice. No use, distribution or reproduction is permitted which does not comply with these terms. 